\title{
Our second year by the numbers
}

\author{
Our annual report of the journal's statistics shows little overall change on gender and geographical diversity, and \\ highlights areas where our editors want to redouble efforts - with help from you.
}

N ature Ecology \& Evolution is now two years old. We celebrate this with our second cartoon cover, this time drawn by Rohan Chakravarty and featuring a selection of the species that have graced our pages during 2018. A year ago, we took a look at some figures from our first year, and we now report on similar figures from our second year. As with last year's numbers, these come with the caveat that they are imperfect, because they have been collated by hand rather than through a formal data-gathering process. The incomplete data and the sample of two years make it inappropriate to subject them to formal statistical analysis. The full figures are available here.

Given the data we have available, we can only look at gender of our authors and reviewers in a binary way, based just on name, and for the larger data sets of reviewers and corresponding authors of submitted manuscripts, this left gender unassigned for up to $25 \%$ of individuals. Of our published research manuscripts, $18 \%$ $(35 / 194)$ had female corresponding authors. This compared with $23 \%(275 / 1,188)$ of the submitted manuscripts and 22\% (58/258) of those sent to peer review. These numbers are alarming, for several reasons. Most obviously, although there appears to be no bias in the proportion of manuscripts with female corresponding authors that we send to peer review, there is potentially a bias in the number that end up being published. A caveat here is that the set of published manuscripts does not necessarily correspond exactly to the sets of submitted and reviewed manuscripts, because the latter are taken from a time window of initial submission (1 May 2017 to 30 April 2018) that is hoped but not guaranteed to represent manuscripts published in 2018. For this reason, we are unable to compare the data sets formally, but we are making it a top priority to guard against any gender biases occurring at the post-review decision stage. We cannot, unfortunately, guard against gender bias introduced by peer reviewers, although we can - and are increasing the gender balance among our reviewers (see below). A second cause for concern is that these proportions are lower than last year, when $24 \%$ of our submitted manuscripts, $27 \%$ of our peer reviewed manuscripts and $27 \%$ of our published manuscripts had female corresponding authors. We need more data to identify any trends definitively, but it is certainly something we will continue to monitor.

For our reviewers and the authors of nonresearch manuscripts, the picture looks more encouraging. Of the 1,134 reviewers whose gender could be assigned with reasonable confidence, 389 (34\%) were women, and we believe the increase from $26 \%$ last year is indicative of our efforts to reach a wider and more diverse pool of reviewers. Of the 114 non-research manuscripts we published, 39 (34\%) had female corresponding authors, which is similar to the $36 \%$ last year. It is worth noting, though, that this proportion is driven mainly by the authorship of News \& Views, our only section that is exclusively commissioned, and of which women make up just over half the corresponding authors (51\%).

Last year, when we looked at the geographical distribution of our corresponding authors and reviewers, we noticed that we received proportionally far more submissions from Asia than we ended up publishing, and we recruited an even lower proportion of peer reviewers from that continent. In an effort to reach out to the research community in Asia, our editorial team attended conferences in China and Japan this year. Nevertheless, the figures for 2018 look very similar: $21 \%$ of submissions versus $9 \%$ of published research manuscripts versus $4 \%$ of both peer reviewers and published non-research manuscripts came from Asia (we were unable this year to obtain geographical data on manuscripts sent for peer review). It is worth noting that these author figures relate only to corresponding authors, and therefore do not capture the full geographical diversity of many research teams. Because many factors play into the difference between the numbers of submitted versus published manuscripts, we cannot guarantee immediate change to that figure, but we are committed to increasing our proportion of reviewers and non-research authors from Asia and other underrepresented regions.

Overall, the figures for geographical distribution (Fig. 1) mirror those from last year. One change we made this year was to look at the numbers from the United Kingdom separately from the rest of Europe. Our main reason for doing this is that we

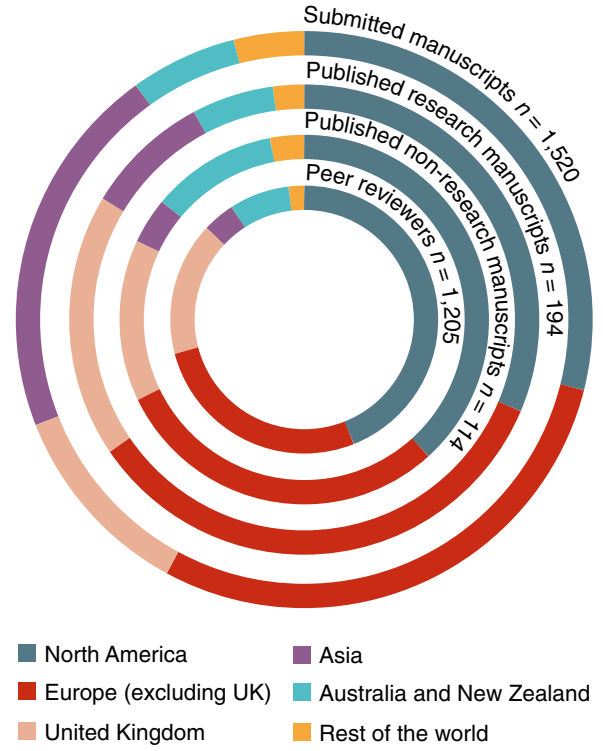

Fig. 1 | Geographical distribution of corresponding authors and reviewers.

are concerned that, as an editorial team that until recently was entirely based in the United Kingdom (we now have one editor in Australia), we might have a bias towards manuscripts from this country. The figures do imply this as a possibility: the United Kingdom accounts for $11 \%$ of our submitted manuscripts but $18 \%$ of our published ones (as well as 16\% of our peer reviewers and $14 \%$ of our non-research manuscripts). There are, of course, other factors that could partially explain this, such as the United Kingdom's historically strong investment in research, and ecology and evolution in particular, or bias in peer reviewer reports. We will keep monitoring these figures, both to aim to prevent any editorial bias and to observe any effect of the current political crisis in the United Kingdom, and probable Brexit, on research output.

One other consequence of separating out the United Kingdom from the rest of Europe is that it allows us an approximate but easy way of estimating the proportion of authors and reviewers based in countries where English is spoken as a first language. Whereas only $46 \%$ of our submitted manuscripts come from such countries, $56 \%$ of our published research output, $63 \%$ of our published non-research output and $67 \%$ of our peer 
reviewers do. As editors, we consider it an important part of our role to level the playing field between native and non-native speakers of English, and we seek to do this through considering initial research submissions on the basis of quality and impact, rather than language proficiency, and through commissioning and assisting non-English speakers to write for the journal.
As we continue our efforts to represent the diverse research community in our pages, we also need your support. A recent Comment highlights the many ways bias manifests in science research and publishing, and calls for the entire community to contribute to confronting this. From our perspective, authors and reviewers can help by considering biases and thinking broadly when suggesting reviewers and deciding who should be a corresponding author. We also encourage research and non-research contributions to the journal from all parts of the community - we are eager to hear from you.

Published online: 19 December 2018 https://doi.org/10.1038/s41559-018-0779-9 